\title{
Assessment of Post Chemotherapy Complications among Hematological Malignancy Patients
}

\author{
Fatma Ahmed Khalifa ${ }^{1}$, Mervat Anwar Abdel-Aziz ${ }^{2}$ \& Khaled Fathi Riad $^{3}$ \\ ${ }^{1}$ Specialist nursing at south Egypt cancer institute. \\ 2. Assistant Professor of Critical \& Emergency Care Nursing, Faculty of Nursing, Assiut University, Egypt. \\ ${ }^{3 .}$ Lecturer of pediatric oncology, South Egypt cancer institute - Assiut University, Egypt.
}

\begin{abstract}
Background: Patients with Hematological malignancy patient need extraordinary nursing precaution for improving the consequence and prevent post chemotherapy complication. Aim: To assess post chemotherapy complications among hematological malignancy patients. Methodology: Design: descriptive research design was utilized in this study. Setting: The study was conducted in intensive care unit at south Egypt cancer institute. Sample: A convenient sample of 60 patients with hematological Malignancy. Tools: Two tools used for data collection, (I): patient assessment tool consisted of patient Socio demographic, clinical data and Physical assessment. (II): Post chemotherapy complications assessment sheet. Results: main sex of the studied patients were male their ages were 24.4 55.04, more than one third of them diagnosed of AML. The main complications were, fever, malaise, fatigue, nausea, and mucositis. Also, the majority suffered from muscle cramps, bone pain, weakness and headache. Conclusion: The main complications were, fever, malise, fatigue, nausea, microsites, muscle cramps, bone pain, weakness and fatigue, headache and site reaction. Recommendation: Keep a standardized nursing guideline for assessing and caring of post chemotherapy complications among the hematological malignancy patients should be available in a written form in the oncology unit.
\end{abstract}

\section{Keywords: Hematological, Malignancy \& Post Chemotherapy Complications}

\section{Introduction}

Hematologic cancer considers a disease that influences blood, lymph nodes and bone marrow. Hematologic cancer address the most regularly happening disease and the subsequent driving reason for disease passing. The most well-known types of hematologic disease are leukemia and lymphoma. Leukemia happens when the bone marrow over produces unusual white platelets, and is characterized by the kind of white platelet influenced; myeloid or lymphocytic. Lymphoa is a disease of the lymphatic framework that outcomes in case it uncontrolled development of threatening white platelets shaping tumors. Lymphoma is ordered into Hodgkin's and non Hodgkin's lymphoma (Jeong et al., 2020).

Anorexia, lymph node (gland) extension, Lumps or stomach distension because of amplified stomach organs, abdominal agony, bone pain, back pain, disarray headaches, visual aggravation, liquid maintenance diminished urination (Sherazi, 2018). Complications of hematological malignancy Include; neutropenia, hemorrhagic complication, anemia and bleeding, acute pulmonary complications because they are in an immune compromised state due to systymic disease or to chemotherapy (pulmonary hemorrhage, edema, leukostasis, and pneumonia) (Jamme et al., 2017).
Cancer management are located according to cancer type including: surgery; chemotherapy; radiation therapy; immunotherapy; and stem cell trance plant; chemotherapy is the use of cytotoxic drugs in the treatment of cancer (Schirrmacher, 2019).

There are many complications of chemotherapy; includes; respiratory, cardio vascular, renal, and nervous system. The cardiovascular harmfulness because of chemotherapy is normal and might be perilous or cause huge morbidities. Chemotherapy drugs will in general harm myocytes, heart valves, vessels and the pericardium. A few chemotherapy medications can cause intense or constant renal disappointment (Oun et al., 2018).

Basic chemotherapy drugs are regularly a reason for tubular and glomerular damage. Chemotherapy can harm any piece of the nervous system. Chemotherapy specialists can cause neurotoxicity, fringe neuropathy, muscle torment, cranial neuropathy, and seizures or intensify previous neurological conditions. Gastrointestinal poisonousness is a typical symptom of most chemotherapy drugs. Signs and indications of gastrointestinal poisonousness incorporates: sickness and spewing, mucositis, nervous system (Malyszko et al., 2020).

Monitor hematocrit, hemoglobin, white blood count and platelet counts. Assessment before and after infusion or injection of chemo therapeutic drug. Safe 
handling practice guidelines cover the following; drug preparation, drug administration, disposal of supplies and unused drugs. Care of patients receiving chemo therapy (e.glinen contamination, patientexcreta). Staff education. Employment practice regarding reproductive issue. Extravasation management (Zhang et al., 2018).

\section{Significant of the study}

Statistical reports of hematological malignancy at intensive care unit at Assiut University (south Egypt cancer Institute) documented that in (2016-2019) 600 cases diagnosed with hematological malignancy. Advancements in the treatment of cancer have improved the prognosis of patients with hematological malignancies, to the extent that treatment is now often given with curative intent. Complications of chemotherapy might occur as a result of off-target' drug effects that associated with high morbidity and a long hospital stay, and is high expensive. Hematological malignancy patient need special nursing care to improve the patient outcomes and prevent early complication. This required, for tumor and management in cancer patients, combination by oncologists and nursing staff working together for optimal care. So, this study aimed to assess post chemotherapy complications among hematological malignancy patients that will be the baseline for building a quality nursing care.

\section{Operational definition:}

Post chemotherapy complications: is the complications that induced in the cancer patients due to receiving chemotherapy on all body systems (short term) within the first 24 hours of the first treatment.

Hematological malignances: are cancers that affect the blood, bone marrow, and lymph nodes. This classification includes various types of leukemia (acute lymphocytic (ALL), chronic lymphocytic (CLL), acute myeloid (AML), chronic myeloid $(\mathrm{CML})$ ), myeloma, and lymphoma (Hodgkin's and non-Hodgkin's (NHL))

Aim of the study:

The aim of the study was to assess post chemotherapy complications among hematological malignancy patients

\section{Research question:}

What are the post chemotherapy complications among hematological malignancy patients?

\section{Subjects and Methods:}

Research design: Descriptive research design was applied.

Setting: it was carried out in the intensive care unit at south Egypt cancer institute
Sample: A convenient sample of sixty (60) patients with hematological Malignancy were included in this study.

\section{Sample size:}

In this study sample size of the studied patients was calculated by using the epi- info program with a confidence level at $95 \%$ and the flow rate of patients 600 cases in a year so the sample was calculated to be 73 patients 13 patients drop out during the data collection, only 60 patients agreed to participate and completed the study period who admitted to the department.

Tools of data collection: The researchers utilized two tools in this study:

Tool (I): patient assessment tool; it was developed by the researcher after go through the literature review.

Part (1): Patient Socio demographic data:

Socio demographic data: patient's code, age, sex, and marital status etc.....

\section{Part (2) - Patient clinical data:}

It used to assess patients' diagnosis, date of admission, length of ICU stay, date of discharge, past medical history, type of chemotherapy, dose, presence and previous transplantation ....etc.

\section{Part (3) - Patient physical assessment include}

- To assess hemodynamic and cardiovascular; temperature, respiration, blood pressure, pulse rate, and mean arterial pressure. Respiratory; rate, depth, pattern, arterial blood gases to assess level of oxygenation, saturation of oxygen measured by pulse oximetry in addition to mode of oxygen therapy.

- In addition to assess the gastrointestinal neuromusculoskeletal, cardio- pulmonary, hematological and urinary systems.

Tool (II): Post chemotherapy complications assessment sheet:

This sheet aimed to assess presence or absence of post chemotherapy complications.

This done through direct observation and filling checklist of the complications among the studied patients.

General condition; (fever, malaise, fatigue)

Gastrointestinal complications (anorexia, nausea, vomiting, mucositis and diarrhea)

Neuro-musculoskeletal complications (muscle cramps, loss of muscle strength, weakness and fatigue, convulsion and headache)

Cardio-pulmonary complications (hypotension, chest pain, myocardial infarction, cough, pulmonary edema, and dyspnea).

Hematological complications (neutropenia, bleeding, erythema, local infusion infection, skin reaction, and rash flushing).

Urinary complications (hematuria). 


\section{Scoring system:}

Presence of the complications $=1$

Absence of the complication $=$ zero

Total degrees $=27$ ranged from zero to 27 degrees

While the patient consider mild $<9$ degree

Moderate from 9 to $<18$ degrees

Sever from $>18$ degrees

Technique for data collection:

Methods:

- The study was conducted throughout the following steps:

- Official permission to conduct the study was obtained from the hospital responsible authorities in the emergency medical unit after illumination the aim and the nature of the current study.

- Development of tools (I and II) after reviewing the related national and international literature.

Tools validity and reliability:

Face vaidity was established by panel of (3) experts in the critical care nursing and critical care medicine fields who reviewed the instruments for clarity, relevance, comprehensiveness, understanding, applicability and easiness for administration while the necessary modification were performed. Then, minor modifications were required. Test reliability of the proposed tool was ascertained with Cronbach's alpha $=0.95$ and 0.89 for tool I and II.

Pilot study:

Supported by $(10 \%=6$ patients $)$ who met the determined criteria for clarity and applicability of the tools' assessment. There was no necessary modification was done then the plot sample was added to the main sample.

\section{Field work:}

Once authorization was arranged to continue with the proposed study researcher initiated data collection.

- Every patient diagnosed with hematological malignancy included in the study.

- The tools were all filled with interviewing patients for explanation the study's purpose and answering the question through 30 minutes for each patient individually.

- The researcher was assessed the studied patients who suffering from hematological malignancy using previous mentioned tools from the first day of admission ( $1^{\text {st }} 24$ hour) to assess the short term complications.

- Patient data were collected by the researchers using (Tool I) to assess:

- Patient's characteristics included patient age, sex, etc... besides, medical data by assessing the medical diagnosis and family history (tool I, part 1) from the patient hospital folder.

- Hemodynamic parameters (tool I, part 2).
- Laboratory investigations results were taken from the patient folder (tool I, part 3).

- Assessment post chemotherapy complications among the studied patients using (Tool II) checked for its presence or absence through daily observation of the patients.

Ethical considerations:

- No risks on the study patients through the research application.

- The researchers obtained an informed consent from the studied patients after explaining the nature and purpose of the study.

- Anonymity and confidentiality were assured.

- During the data collection, patients' privacy was considered.

- Patients had the right to withdraw from the study any time without any rational.

Statistical analysis:

The collected data were entered in the statistical package for (SPSS) version (23) then analyzed and tabulated. 


\section{Results:}

Table (1): Distribution of the studied patients' socio-demographic data $(\mathbf{n} .=60)$

\begin{tabular}{|c|c|c|}
\hline & \multicolumn{2}{|c|}{$\overline{\text { Study }}$} \\
\hline & $n_{.}=60$ & $\%$ \\
\hline \multicolumn{3}{|l|}{ Sex } \\
\hline Male & 38 & 63.3 \\
\hline Female & 22 & 36.7 \\
\hline \multicolumn{3}{|l|}{ Age } \\
\hline $18<28$ & 38 & 63.3 \\
\hline $28<38$ & 10 & 16.7 \\
\hline $38<48$ & 10 & 16.7 \\
\hline$\geq 48$ & 2 & 3.3 \\
\hline Mean \pm SD & \multicolumn{2}{|c|}{$24.4 \pm 5.04$ years old } \\
\hline Diagnosis & & \\
\hline Acute Myeloid Leukemia & 24 & 40.0 \\
\hline Acute Lymphatic Leukemia & 22 & 36.7 \\
\hline Non Hodgkin & 14 & 23.3 \\
\hline
\end{tabular}

Table (2): Mean \pm SD of studied patients regarding the level of hemodynamic status $(\mathbf{n} .=60)$.

\begin{tabular}{|l|c|}
\hline \multicolumn{1}{|c|}{ Hemodynamic status } & On admission (1 $\mathbf{1}^{\text {st }} \mathbf{~ h o u r s )}$ \\
\hline Temperature & $37.78 \pm 0.33$ \\
\hline Pulse & $107.76 \pm 15.82$ \\
\hline Respiration & $18.64 \pm 3.43$ \\
\hline SBB & $110 \pm 15.77$ \\
\hline DBB & $67.34 \pm 7.7$ \\
\hline
\end{tabular}

Chi square test for qualitative data

Table (3): Mean \pm SD of laboratory tests among the studied patients (n. $=60)$.

\begin{tabular}{|l|c|}
\hline \multicolumn{1}{|c|}{ Hemodynamic status } & On admission (1 ${ }^{\text {st }} \mathbf{2 4}$ hours $)$ \\
\hline paO2 & $99.57 \pm 0.4$ \\
\hline Hemoglobin & $10.5 \pm 1.28$ \\
\hline WBC & $7.27 \pm 2.13$ \\
\hline RBC & $3.97 \pm 0.32$ \\
\hline neutrophil count & $1.5 \pm 0.63$ \\
\hline Platelets & $125.93 \pm 25.93$ \\
\hline Serum sodium $(\mathrm{Na})$ & $135.47 \pm 0.78$ \\
\hline Serum potassium $(\mathrm{k})$ & $3.73 \pm 0.52$ \\
\hline Serum calcium & $8.7 \pm 0.7$ \\
\hline Urea & $15.47 \pm 6.8$ \\
\hline Creatinine & $0.33 \pm 0.55$ \\
\hline
\end{tabular}

Chi square test for qualitative data

Table (4): Distribution of post chemotherapy complications (general condition, gastrointestinal and neuromusculoskeletal) among the studied patients $(n .=60)$.

\begin{tabular}{|c|c|c|}
\hline \multirow{2}{*}{ Post chemotherapy complications } & \multicolumn{2}{|c|}{ On admission ( $1^{\text {st }} 24$ hours $)$} \\
\hline & n. & $\%$ \\
\hline \multicolumn{3}{|l|}{ General condition } \\
\hline Fatigue & 46 & 76.7 \\
\hline Fever & 38 & 63.3 \\
\hline Malise & 36 & 60.0 \\
\hline \multicolumn{3}{|l|}{ Gastrointestinal complications } \\
\hline Mucositis & 48 & 80.0 \\
\hline Nausea & 40 & 66.7 \\
\hline Anorexia & 28 & 46.7 \\
\hline Diarrhea & 26 & 43.3 \\
\hline Vomiting & 22 & 36.7 \\
\hline
\end{tabular}




\begin{tabular}{|l|c|c|}
\hline \multicolumn{2}{|c|}{ Post chemotherapy complications } & \multicolumn{2}{c|}{ On admission (1 ${ }^{\text {st }} \mathbf{2 4}$ hours) } \\
\cline { 2 - 3 } & \multicolumn{2}{c|}{ n. } \\
\hline Neuro-musculoskeletal complications & 56 & 93.3 \\
\hline weakness and fatigue & 48 & 80.0 \\
\hline Headache & 38 & 63.3 \\
\hline bone pain & 36 & 60.0 \\
\hline Muscle cramps & 28 & 46.7 \\
\hline Loss of muscle strength & 22 & 36.7 \\
\hline Convulsion & & \\
\hline
\end{tabular}

Chi square test for qualitative data

Table (5): Distribution of post chemotherapy Complications (cardio- pulmonary, hematological and urinary) among the studied patients (n. $=60)$.

\begin{tabular}{|c|c|c|}
\hline \multirow{2}{*}{ Complications } & \multicolumn{2}{|c|}{ On admission ( $1^{\text {st }} 24$ hours $)$} \\
\hline & n. & $\%$ \\
\hline \multicolumn{3}{|l|}{ Cardio-Pulmonary complications } \\
\hline Cough & 34 & 56.7 \\
\hline Dyspnea & 28 & 46.7 \\
\hline Chest pain & 22 & 36.7 \\
\hline Hypotension & 10 & 16.7 \\
\hline Myocardial infraction & 8 & 13.3 \\
\hline Pulmonary edema & 8 & 13.3 \\
\hline \multicolumn{3}{|l|}{ Hematological complications } \\
\hline Site reaction & 34 & 56.7 \\
\hline Local infusion reaction & 32 & 53.3 \\
\hline Skin rash & 28 & 46.7 \\
\hline Flushing & 28 & 46.7 \\
\hline Neutropenia & 22 & 36.7 \\
\hline Bleeding & 22 & 36.7 \\
\hline Erythema & 18 & 30.0 \\
\hline \multicolumn{3}{|l|}{ Urinary complications } \\
\hline Hematuria & 16 & 26.7 \\
\hline
\end{tabular}

Chi square test for qualitative data

Table (1): Showed that the main sex of the studied patients with heamatolgical malignancy and receiving chemotherapy was male their ages were $24.4 \pm 5.04$ years old, more than one third of them diagnosed of Acute Lymphatic Leukemia from $9.36 \pm 5.03$ years.

Table (2): Revealed that comparison between $1^{\text {st }} 24$ hours of admission regarding the level of hemodynamic status of the studied patients it found no change in the patients' temperature $37.78 \pm 0.33{ }^{\circ} \mathrm{C}$ and respiration $(18.64 \pm 3.43)$. While the pulse rate slightly increased from $(107.76 \pm 15.82)$ to $(116.64 \pm 70.15) \mathrm{b} / \mathrm{m}$ and minimal decrease in SBB and DBB from $(110 \pm 15.77)$ and $(67.34 \pm 7.7)$ to $(109.48 \pm 13.97)$ and $(65.34 \pm 6.87) \mathrm{mm} \mathrm{hg}$.

Table (3): Reported that the laboratory investigation levels of the studied patients at admission were Pao2 level that within normal range $(99.57 \pm 0.4)$. Hemoglobin level were $10.5 \pm 1.28 \mathrm{mg}$.

Table (4): Concluded that regarding post chemotherapy complications (general, gastrointestinal and neuro-musculoskeletal) on admission $\left(1^{\text {st }} 24\right.$ hours) majority had fatigue $(76.7 \%)$ regarding gastrointestinal complications majority had microsites (80\%) regarding neuro-musculoskeletal complications most of them suffered from weakness and fatigue $(93.3 \%)$.

Table (5): Concluded that the main complications were, cough, dyspnea (56.7 and 46.7\%), site reaction, local infusion, skin rash and flushing $(56.7,53.3,46.7$ and $46.7 \%$ respectively).

\section{Discussion:}

Hematological malignancies consider a heterogeneous gathering of sicknesses portrayed by uncontrolled harmful expansion of hematopoietic cells. The recurrence of it has expanded with a great difference between the developed and developing nations (Fuji et al., 2020). So, this study aimed to assess post chemotherapy complications among hematological malignancy patients regarding the demographic data of the studied patients our results have shown that AML was presented of two fifth of the studied patients. This matched with Hussein et al., (2019) who that leukemia was the most widely recognized introduced Hematological malignancies in 
Assiut, Upper Egypt. In addition, Tharwat et al., (2019) found acute myeloid leukemia (AML) was the furthermost the most type then NHL preponderates in lymphoma.

Our study found that majority of our study was males with middle ages. this not match with study of Mohammed et al., (2017) which conducted among Sudanian, discovered the greatest number of cases were found in the age gathering of 45-64 years and in both sex, leukemia had a higher recurrence than lymphoma among the announced common cancer sites.

But, this agreed with Hossain et al., (2017), found among Bangladesh leukemic patients, were more than three times higher contrasted amongst males. In acute leukemia, hypothesis of male risk stays an unexplained fact (Ugai et al., 2017). Laboni, (2020) found that in malignancy diseased patients, being male is among the normal danger factors. Interestingly, the current investigation showed that female contrasted and men had a fundamentally higher danger for creating HMs and all its subtypes aside from lymphoma.

This concurs with Shysh et al., (2018) who found that acute myeloid leukemia is generally basic in older people and influences males more than females. Bron et al., (2017) distinguished that the significant predictors of HMs as generally speaking and its subtypes, expanding age was a significant risk factor for all HMs with all its subtypes. There is proof of relationship among aging and cancer especially HMs. This could be credited to segment move, delayed openness to cancer-causing agents and persistent antigenic incitement and age-went with changes in hereditary qualities and invulnerability. Duarte et al., (2018) detailed that subsequent hereditary harm changes don't avoid hematopoietic stem cells. Gender orientation is a significant contributing variable that impacts the danger factors for illnesses (Humphries et al., 2017).

An alternate example was noticed, where lymphoma extraordinarily NHL was the most every now and again introduced HM as announced by the US Cancer Fact and Figures (Siegel et al., 2017). The distinction in the appropriation of HMs between both Upper and Lower Egypt could be ascribed to the variety in hazard factors that should have been researched in future. As to recognized huge indicators of HMs as in general and its subtypes in this investigation, the high age the higher danger factor for all types hematological cancers.

This not concurred with Kuznetsova et al., (2017) who referenced that the average age of acute myeloid leukemia was $48.9 \pm 18.3$ years old. Medinger \& Passweg, (2018) checked that AML is currently restored in around $35 \%-40 \%$ of patients less than 60 years old.

The resent study found that regarding the level of hemodynamic status of the studied patients found the patients' temperature and respiration not affected. While the pulse rate slightly increased and minimal decrease in SBB and $\mathrm{DBB}$. The study changes in Pao2 level that within the normal range. This concluded by Biskup et al., (2017) found among ICU hematological malignancies patients suffered from neurological disappointment, which considered normal and regularly fetal. Independent predictors of higher mortality were poor health status, in adequate hemodynamic and respiratory prestige.

This result contrast with Verlinden et al., (2019) who exhibited that fever was altogether connected with hematological malignancy and because of contamination couldn't be dependably separated from fever not because of disease by either the stature or character of the fever bend, or by checking without a doubt the quantity of develop leukocytes.

Our study found that post chemotherapy complication (general, gastrointestinal and neuro-musculoskeletal) on admission majority had fatigue regarding gastrointestinal complications majority had microsites regarding neuro-musculoskeletal complications most of them suffered from weakness and fatigue.

This match with Avancini et al., (2020) who found that nausea and vomiting considered a typical clinical issue in the high level malignancy patient. The etiology might be identified with therapy (chemotherapy, radiation, medical procedure) or nontherapy clinical issues identified with the high level disease. Also, Navari, (2020) annoyed that an exceptionally itemized starting appraisal of nausea/vomiting is shown including recurrence, term, power, related exercises, and the presence of anorexia or cachexia and is fundamental to decide a particular etiology which may permit a possibly explicit effective intervention.

This not match with Chowdhri et al., (2018) the greater part of the considered patients experienced bleeding the leftover example experienced ulceration were the most well-known beginning determination indication of leukemia.

Hospital mortality increased among hematological malignancy patients, with regards to the almost selective event of CNS inclusion in the forceful types of this illness, which are additionally connected with high mortality (Probst et al., This match with Falk et al., (2017) who found that Cancer-induced bone pain (CIBP) includes both neuropathic and provocative torment pathways, related with tumor, stroma, and adjoining tissues, including fringe and central nerves. Concerning the muscle weakness McGlory et al., (2019) reported that the skeletal muscle misfortune 
because of cachexia results from diminished protein anabolism, expanded proteolysis, or a mix of both.

The major proteolytic pathways in skeletal muscle are; the calcium-activated calpains I and II, which primarily cause tissue injury, necrosis and autolysis. The ATP-dependent ubiquitin proteasome proteolytic pathway, which the whole thing with the calpain system to corrupt myofilaments. This pathway assumes a dominating part in debasement of myofibrillar proteins particularly in patients with a weight reduction of $>10 \%$ (Milgrom et al., 2017).

Regarding to Waning, (2017) study found Cancer cachexia is a complex metabolic condition portrayed by skeletal muscle squandering (with or without fat misfortune), anemia, decreased caloric admission, and changed invulnerable capacity, which adds to expanded incapacity, weariness, lessened quality of life (QoL), and diminished endurance.

The current study found that the main complications were, cough, dyspnea, site reaction, local infusion, skin rash and flushing.

This agreed with Booth \& Johnson, (2019) who announced that, dyspnea, any attention to breathing, can truly influence personal satisfaction in in cancer patients. In this respect Baker et al., (2017) added that, dyspnea may restrict movement to the degree that even the smallest effort will accelerate shortness of breath. Additionally, Lippiett et al., (2019) in the terminal phase of ailment, dread of suffocation may address the most alarming indication for cancer patients. Regardless of its commonness and its seriousness, Pisani et al., (2018) recorded that dyspnea in malignant growth patients has gotten shockingly little consideration in research or in the literature.

In this discovering, Matynia et al., (2018) detailed that acute respiratory inconveniences happen habitually during the early phase of acute myeloid leukemia (AML) yet data on the most serious structure. Moreau et al., (2017) affirmed this outcome when reflectively investigated clinical and research facility information from hundred patients conceded to a clinical ICU inside ten days after a determination of hematological threat recommending a part as a preventive therapy for cancer patients with non-infectious pulmonary immersion.

In this respect, Brown et al., (2017) originated that majority of hematological cancer with mild bleeding is related with the danger of serious dying. This is a significant finding for a few reasons. It offers help for the utilization of mild bleeding as a study outcome measure.

Numerous physicians feel that the solitary bleeding that is really of result is seeping of evaluation three or four in seriousness. Nonetheless, if grade one and two drains are affirmed to be prescient of severe bleeding to be utilized as clinically pertinent results (Abdel Haady et al., 2019).

Misirliog et al., (2017) referenced that; diseases specially anemia consider the major causes of leukemic patients mortality. Anemia is a typical difficulty in patients with hematologic malignancies, and is brought about by an assortment of components, including neoplastic cell invasion into the bone marrow, hemolysis, nourishing lacks, and deformities in erythropoiesis because of the actual illness or cytotoxic treatment (Gavriilaki et al., 2019).

Since, Busti et al., (2018) archived that anemia is so common in hematologic harm patients, its therapy should be an indispensable piece of sickness the executives, to improve personal satisfaction and to perhaps expand likely endurance. Clinical examinations have shown that viably treating pallor and expanding hemoglobin levels utilizing recombinant human erythropoietin significantly affects bonding prerequisites and quality of life.

\section{Conclusion:}

It was concluded that;

- The laboratory investigation levels of the studied patients were within normal ranges.

- Regarding post chemotherapy complications (general, gastrointestinal and neuromusculoskeletal) on admission ( $1^{\text {st }} 24$ hours) majority of the studied patients had fatigue (76.7\%).

- Regarding gastrointestinal complications majority of the studied patients had microsites (80\%) regarding neuro-musculoskeletal complications most of them suffered from weakness and fatigue $(93.3 \%)$.

- The main complications were, cough, dyspnea (56.7 and 46.7\%), site reaction, local infusion, skin rash and flushing (56.7, 53.3, 46.7 and $46.7 \%$ respectively).

\section{Recommendations:}

Based on the findings of the present study, the following recommendations are derived:

- Keep a standardized nursing guideline for assessing and caring of post chemotherapy complications among the hematological malignancy patients should be available in a written form in the oncology unit.

- Duplicate of the study on a greater prospect sample acquired from different geographical areas. 
References

- Abdel Haady, M., Shawer, O., \& Abdel-Aziz, M. (2019): Effect of Implementing Nursing Instructions on Minimizing Bleeding Among Patients with Acute Myeloid Leukemia. Assiut Scientific Nursing Journal, 7(19), 186-194.

- Avancini, A., Trestini, I., Tregnago, D., Cavallo, A., Bragato, M., Bonaiuto, C., \& Pilotto, S. (2020): Multidisciplinary lifestyle intervention to manage pancreatic cancer-related cachexia: a case report. Future Science OA, (0), FSO659.

- Baker, K., DeSanto-Madeya, S., \& Banzett, R. (2017): Routine dyspnea assessment and documentation: Nurses' experience yields wide acceptance. BMC nursing, 16(1), 3.

- Biskup, E., Cai, F., Vetter, M., \& Marsch, S. (2017): Oncological patients in the intensive care unit: prognosis, decision-making, therapies and endof-life care. Swiss Medical Weekly, 147(3132.

- Booth, S., \& Johnson, M. (2019): Improving the quality of life of people with advanced respiratory disease and severe breathlessness. Breathe, 15(3), 198-215.

- Brown, H., Andersson, T., Björkholm, M., Dickman, P., Lambert, P., \& Derolf, Å. (2017): Continued improvement in survival of acute myeloid leukemia patients: an application of the loss in expectation of life. Blood cancer journal, 6(2), e390.

- Bron D, Ades L, Fulop T, Goede V, Stauder R. (2017): Aging and blood disorders: new perspectives, new challenges. Haematologica; 100:415-417.

- Busti, F., Marchi, G., Ugolini, S., Castagna, A., \& Girelli, D. (2018): Anemia and iron deficiency in cancer patients: role of iron replacement therapy. Pharmaceuticals, 11(4), 94.

- Duarte, D., Hawkins, E., Akinduro, O., Ang, H., De Filippo, K., Kong, I., \& McLean, C. (2018): Inhibition of endosteal vascular niche remodeling rescues hematopoietic stem cell loss in AML. Cell stem cell, 22(1), 64-77.

- Falk, S., Bannister, K., Dickenson, AH, (2017): Cancer pain physiology. Br J Pain. ; 8(4):154-62.

- Fuji, S., Nagler, A., Mohty, M., Savani, B., \& Shouval, R. (2020): Decision-analytic modeling as a tool for selecting optimal therapy incorporating hematopoietic stem cell transplantation in patients with hematological malignancy. Bone marrow transplantation, 1-9.

- Gavriilaki, E., Gkaliagkousi, E., Grigoriadis, S., Anyfanti, P., Douma, S., \& Anagnostopoulos, A. (2019): Hypertension in hematologic malignancies and hematopoietic cell transplantation: an emerging issue with the introduction of novel treatments. Blood reviews, 35, 51-58.
- Hossain MS., Iqbal MS., Khan MA., Rabbani MG, Khatun H, \& Munira S. (2017): Diagnosed hematological malignancies in Bangladesh-a retrospective analysis of over 5000 cases from 10 specialized hospitals. BMC Cancer; 14:438.

- Humphries, K., Izadnegadar, M., Sedlak, T., Saw, J., Johnston, N., Schenck-Gustafsson, K., \& Wei, J. (2017): Sex differences in cardiovascular disease-impact on care and outcomes. Frontiers in neuroendocrinology, 46, 46.

- Hussein, S., Mohamed, D., \& Hafez, R. (2019): Risk factors of hematological malignancies in Upper Egypt: a case-control study. The Egyptian Journal of Internal Medicine, 31(2), 171.

- Jamme, M., Daviaud, F., Charpentier, J., Marin, N., Thy, M., Hourmant, Y., \& Pène, F. (2017): Time course of septic shock in immunocompromised and nonimmunocompromised patients. Critical care medicine, 45(12), 2031-2039.

- Jeong, S., Choi, T., Kim, D., Han, K., Kim, S., Rhee, S., \& Shin, D. (2020): Association between high-density lipoprotein cholesterol level and risk of hematologic malignancy. Leukemia, 1-9.

- Kuznetsova, I., Labutina, E., \& Hunter, N. (2017): Radiation risks of leukemia, lymphoma and multiple myeloma incidence in the Mayak cohort: 1948-2004. PloS one, 11(9), e0162710.

- Laboni, S. (2020): Urinary Incontinence among Parkinson's Disease Patients in Alberta: Incidence and Risk Predictors, 6(1), 12:20.

- Lippiett, K., Richardson, A., Myall, M., Cummings, A., \& May, C. (2019): Patients and informal caregivers' experiences of burden of treatment in lung cancer and chronic obstructive pulmonary disease (COPD): a systematic review and synthesis of qualitative research. BMJ open, 9(2).

- Malyszko, J., Tesarova, P., Capasso, G., \& Capasso, A. (2020): The link between kidney disease and cancer: complications and treatment. The Lancet, 396(10246), 277-287.

- Matynia, A., Perkins, S., \& Li, D. (2018): Lymphomatoid granulomatosis in a 14-year-old boy with trisomy 21 and history of B-lymphoblastic leukemia/lymphoma. Fetal and pediatric pathology, 37(1), 7-14.

- McGlory, C., Calder, P., \& Nunes, E. (2019): The Influence of Omega-3 Fatty Acids on Skeletal Muscle Protein Turnover in Health and Disease. Frontiers in nutrition, 6, 144.

- Medinger, M., \& Passweg, J. (2018): What the internist should know about stem cell transplant in the elderly patient. European journal of internal medicine, 58, 43-47.

- Milgrom, D., Lad, N., Koniaris, L., \& Zimmers, T. (2017): Bone pain and muscle weakness in 
cancer patients. Current osteoporosis reports, 15(2), 76-87.

- Mohammed, ME., Hassan AM., Abdelhadi HA, Elsadig, MG., Adam DM., Elmamoun K., (2017): Burden and Pattern of Cancer in the Sudan, 20002006.

- Moreau, A., Lengline, E., Seguin, A., Lemiale, V., Canet, E., Raffoux, E., \& Azoulay, E. (2017): Respiratory events at the earliest phase of acute myeloid leukemia. Leukemia \& lymphoma, 55(11), 2556-2563.

- Navari, R. (2020): Nausea and Vomiting in Advanced Cancer. Current Treatment Options in Oncology, 21(2), 14Chowdhri, K., Tandon, S., Lamba, A. K., \& Faraz, F. (2018): Leukemic gingival enlargement: A case report and review of literature. Journal of oral and maxillofacial pathology: JOMFP, 22(Suppl 1), S77.

- Oun, R., Moussa, Y., \& Wheate, N. (2018): The side effects of platinum-based chemotherapy drugs: a review for chemists. Dalton transactions, 47(19), 6645-6653.

- Pisani, L., Hill, N., Pacilli, A., Polastri, M., \& Nava, S. (2018): Management of dyspnea in the terminally ill. Chest, 154(4), 925-934.

- Probst, L., Schalk, E., Liebregts, T., Zeremski, V., Tzalavras, A., von Bergwelt-Baildon, M., \& Eichenauer, D. (2019): Prognostic accuracy of SOFA, qSOFA and SIRS criteria in hematological cancer patients: a retrospective multicenter study. Journal of Intensive Care, 7(1), 41.

- Tharwat, Lt., Coll SM., \& Rahid AD., (2019): "Hematological Malignancies Pattern among Bangladeshi Adults." leukemia (ALL) 5: 6.

- Schirrmacher, V. (2019): From chemotherapy to biological therapy: A review of novel concepts to reduce the side effects of systemic cancer treatment. International journal of oncology, 54(2), 407-419.

- Sherazi, M. (2018): History Overview: General History Hematology. The Objective Structured Clinical Examination Review, 411.

- Shysh, A., Nguyen, L., Guo, M., Vaska, M., Naugler, C., \& Rashid-Kolvear, F. (2018): The incidence of acute myeloid leukemia in Calgary, Alberta, Canada: a retrospective cohort study. BMC public health, 18(1), 94.

- Siegel RL, Miller KD, \& Jemal A. (2017): Cancer statistics, CA Cancer J Clin; 66:7-30

- Ugai T, Matsuo K, Sawada N, Iwasaki M, Yamaji T, Shimazu T. (2017): Smoking and subsequent risk of leukemia in Japan: The Japan Public Health Centerbased Prospective Study. J Epidemiol 2017; 27:305-310.
- Verlinden, A., De Vroey, V., Goossens, H., Roelant, E., Van De Velde, A. L., Berneman, Z. N., \& Gadisseur, A. (2019): Comparison of the power of procalcitonin and c-reactive protein to discriminate between different aetiologies of fever in prolonged profound neutropenia: a single-centre prospective observational study. Mediterranean journal of hematology and infectious diseases, 11(1).

- Waning D.L., (2017): Cancer-associated muscle weakness: What's bone got to do with it? Bonekey Rep.; 4:691.

- Zhang, F., Stephan, S., Ene, C., Smith, T., Holland, E., \& Stephan, M. (2018): Nanoparticles that reshape the tumor milieu create a therapeutic window for effective T-cell therapy in solid malignancies. Cancer research, 78(13), 3718-3730. 